\title{
Improved Photoelectrochemical Performance of GaN Nanopillar Photoanodes
}

Parvathala Reddy Narangari $^{\mathrm{a}^{*}}$, Siva Krishna Karuturi ${ }^{\mathrm{a}^{*}}$, Mykhaylo Lysevych ${ }^{\mathrm{b}}$, Hark Hoe Tan ${ }^{\mathrm{a}}$, and Chennupati Jagadish ${ }^{\mathrm{a}}$

${ }^{a}$ Department of Electronic Materials Engineering, Research School of Physics and Engineering, The Australian National University, Canberra, ACT 2601, Australia

${ }^{\mathrm{b}}$ Australian National Fabrication Facility, Research School of Physics and Engineering, The Australian National University, Canberra, ACT 2601, Australia

Corresponding author: parvathala.narangari@anu.edu.au; siva.karuturi@anu.edu.au

\begin{abstract}
In this work, we report on the photoelectrochemical (PEC) investigation of n-GaN nanopillar (NP) photoanodes fabricated using metal organic chemical vapour deposition and topdown approach. Substantial improvement in photocurrents is observed for GaN NP photoanodes compared to their planar counterparts. The role of carrier concentration and NP dimensions on the PEC performance of NP photoanodes is further elucidated. Photocurrent density is almost doubled for doped NP photoanodes whereas no improvement is noticed for undoped NP photoanodes. While the diameter of GaN NP is found to influence the onset potential, carrier concentration is found to affect both the onset and overpotential of the electrodes. Optical and electrochemical impedance spectroscopy characterizations are utilised to further explain the PEC results of NP photoanodes. Finally, improvement in the photostability of NP photoanodes with the addition of $\mathrm{NiO}$ as a co-catalyst is investigated.
\end{abstract}

Keywords: GaN, Nanopillars, Photoanodes

\section{Introduction:}

Generation of hydrogen fuel by splitting water using renewable solar energy is a highly desirable and exciting prospect for the sustainable development of human race.[1-3] III-V nitrides have recently been gaining considerable research interest in solar water splitting owing to their outstanding properties.[4-8] The advantages of nitride alloys include tunable bandgap, high crystal quality and stability against photo and chemical corrosion. For example, the band gap of $\operatorname{In}_{\mathrm{x}} \mathrm{Ga}_{1}$ ${ }_{x} \mathrm{~N}$ alloys can be tuned from 3.4 to $0.7 \mathrm{eV}$ depending on the In composition. This facilitates the manipulation of light absorption across the entire solar region for efficient solar water splitting 
while straddling the water oxidation and reduction potentials.[9] Typically, GaN layer is grown epitaxially in single crystal quality which is highly beneficial for efficient charge transfer. Moreover, the strong ionic bonding nature of III-nitride alloys helps in stabilizing the photoelectrodes against photo-corrosion. All these superior properties of III-nitrides make them excellent candidate materials for solar water splitting applications.

In addition, nanostructures offer great potential for solar water splitting over their planar counterparts. Nanostructures possess several essential attributes towards achieving efficient water splitting such as enhanced light absorption, reduced carrier transfer lengths, large surface to facilitate efficient charge transfer at semiconductor/electrolyte interface and enlarged depletion area at the nanostructured surface that drives the charge separation efficiently. [10-15] GaN nanostructures can be synthesized via bottom-up and top-down approaches. In the bottom-up approach, nanostructures are grown either by selective area epitaxial growth or vapour-liquid-solid growth $[16,17]$. In the top-down approach, nanopillars (NPs) are formed by reactive ion etching of patterned GaN epilayers.[18-21] Controlling the morphology and doping of NPs is a main setback for bottom-up approaches.[22] On the other hand, NPs fabricated using the top-down approach exhibit controlled morphology with uniform doping as it allows the use of high quality GaN epilayer grown using well-matured planar growth technology wherein doping concentration is precisely controlled. The morphology of the NPs can be controlled by well-established lithography techniques or random masking techniques. Self-assembled random mask cost is effective technique for produce the wafer-scale etch mask.[23, 24] In addition, the density and size of the NP can be controlled by simply varying the thickness of Ni film. As a result, top-down approach produces nanostructures in a cost effective manner at the wafer-scale level. Despite these merits, there are very few reports on the photoelectrochemical (PEC) studies of GaN NPs fabricated using top-down approach.

Carrier concentration and dimensions of nanostructures are important parameters towards realizing efficient water splitting. There are considerable number of research reports on nitridebased PEC water splitting [25-31] and overall direct water splitting. [32-35] However, there are very few studies focused on the influence of carrier concentration and NP dimensions on solar water splitting. Therefore, it is critical to understand the dependence of PEC performance on NP dimensions and carrier concentration. J. Benton et al. [36] reported significant enhancement of 
photocurrent and hydrogen generation for n-GaN/MQW(InGaN/GaN)/p-GaN NPs, fabricated by the top-down process, compared with their planar counterparts. However, the structure applied in their work includes a p-n junction and InGaN multiple quantum wells, which complicate the PEC analysis of GaN NPs. W. J. Tseng et al. [37, 38] investigated the influence of length of the NPs and surface damage to NPs that occurred during the dry etching on the PEC performance of NP photoanodes, however, the photocurrents were significantly low. Nanowires grown in the bottomup approaches exhibit varying doping concentration along the nanowire length. This makes it difficult to understand the influence of carrier concentration on the PEC performance of NP photoanodes. Therefore, top-down approach is an ideal method for fabricating NPs to investigate the influence of carrier concentration and NP dimensions on the PEC performance of NP photoanodes.

In this work, we report on the fabrication of n-GaN NP photoanodes using the top-down approach and the investigation of the influence of carrier concentration and NP dimensions on their PEC performance. Self-assembled random-mask technique was used for producing etch mask. Impedance and optical spectroscopy measurements were carried out to explain the PEC performance of NP photoanodes. The dependence of depletion layer thickness in nanostructures on carrier concentration and NP dimensions is used to explain the shift in anodic potential of the NPs with changing dimensions and carrier concentrations.

\section{Experimental}

For the fabrication of GaN NPs, $2 \mu \mathrm{m}$-thick GaN epitaxial film with controlled carrier concentration was first grown on sapphire wafer using metal organic chemical vapour deposition (MOCVD). Carrier concentration of the GaN epilayers was adjusted by varying the silane flow during the growth. GaN NPs were fabricated from the epilayers using inductively coupled plasmareactive ion etching (ICP-RIE) and self-assembled random nano-mask techniques.[24] $\mathrm{SiO}_{2} / \mathrm{Ni}$ mask was used as an etch mask for the fabrication of GaN NPs. For the ICP-RIE etching of $\mathrm{SiO}_{2}$, $40 \mathrm{sccm}$ of $\mathrm{CHF}_{3}$ gas was passed through the ICP-RIE chamber that was maintained at $20{ }^{\circ} \mathrm{C}$ electrode temperature, $10 \mathrm{mT}$ pressure and 30/1000 watt RF/ICP powers. On the other hand, GaN ICP-RIE etching has been carried out at $60{ }^{\circ} \mathrm{C}$ electrode temperature by passing 25/2/5 sccm of $\mathrm{Cl}_{2} / \mathrm{H}_{2} / \mathrm{Ar}$ gases at a chamber pressure of $4 \mathrm{mT}$ and 200/1000 watt RF/ICP powers. 
$\mathrm{NiO}$ nanoparticle co-catalyst decoration on the surface of GaN NPs was achieved by depositing $5 \mathrm{~nm}$ Ni film on the GaN NPs using e-beam evaporation (E-beam, Temescal BJD2000). This Ni film coated NPs were furnace annealed at $550{ }^{\circ} \mathrm{C}$ in air for 30 min to convert the Ni film into NiO nano-islands. Field emission scanning electron microscopy (FEI Helios Nanolab 600) was employed to investigate the morphology of GaN NPs and NiO-coated NPs. Perkin Elmer spectrophotometer equipped with an integrating sphere was used to characterize the diffuse reflectance of planar GaN and GaN NPs.

To measure the PEC properties of planar and NPs photoanodes Ni/Au (30/150nm) metal contacts were deposited onto the planar and NP GaN samples. The contacts were then subjected to rapid thermal annealing at $400{ }^{\circ} \mathrm{C}$ in argon atmosphere for $5 \mathrm{~min}$. PEC measurements of these photoanodes were carried out under a solar simulator (Newport) fitted with air mass 1.5 filters in $1 \mathrm{M} \mathrm{NaOH}$ electrolyte solution in a quartz cell consisting of the GaN working electrode, a Pt counter electrode and a $\mathrm{Ag} / \mathrm{AgCl}$ reference electrode.

\section{Results and Discussion}

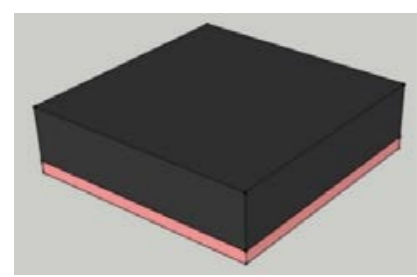

GaN

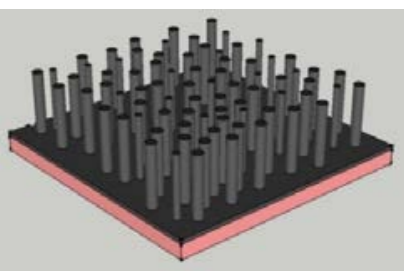

Mask removal

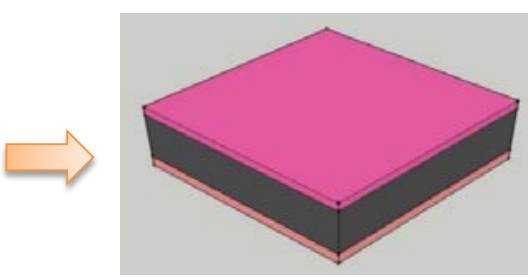

PECVD of $500 \mathrm{~nm} \mathrm{SiO}$

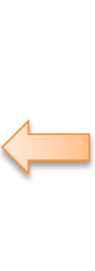

ICP etching of $\mathrm{SiO}_{2}$ and $\mathrm{GaN}$

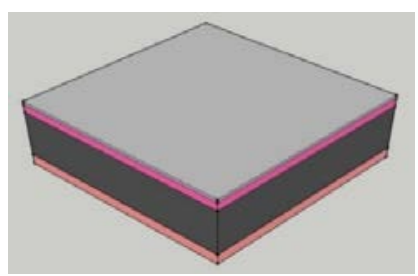

E-beam deposition of $\mathrm{Ni}$
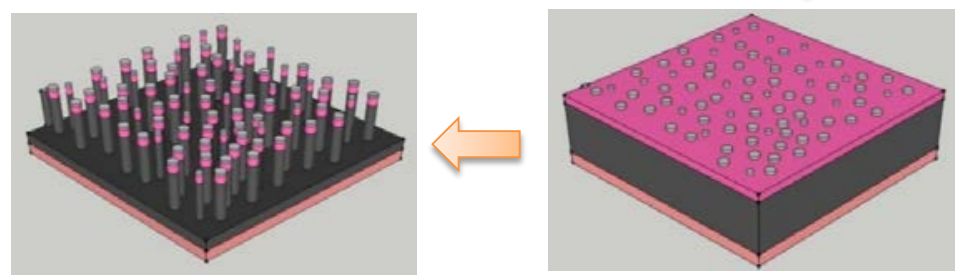

RTA annealing

Fig 1. Illustration of the processing steps involved in the fabrication of GaN NPs using top-down approach.

Fig 1 shows the schematic illustration of top-down fabrication of GaN NPs. GaN nanopillars were fabricated by ICP-RIE etching of $\mathrm{SiO}_{2} / \mathrm{Ni}$ masked n-doped and undoped $\mathrm{GaN}$ wafers as illustrated 
in Fig $1 . \mathrm{SiO}_{2} / \mathrm{Ni}$ hard mask was obtained using plasma enhanced chemical vapour deposition (Oxford Plasmalab 100) of $500 \mathrm{~nm}$-thick $\mathrm{SiO}_{2}$ film on the $\mathrm{GaN}$ wafers followed by electron beam evaporation of 5-15 nm thick nickel film. The Ni film was converted into nano-islands by rapid thermal annealing at $900{ }^{\circ} \mathrm{C}$ for 2 minutes in Ar atmosphere. Next, the $\mathrm{SiO}_{2} / \mathrm{Ni}$ random nanopattern was created by ICP-RIE (Versaline LL) etching of $\mathrm{SiO}_{2}$ surrounding the nickel islands. The $\mathrm{SiO}_{2} / \mathrm{Ni}$ random mask was then used to selectively etch $\mathrm{GaN}$ and produce GaN NPs using ICP-RIE. After etching, GaN NPs were dipped in HF solution to remove the leftover $\mathrm{SiO}_{2} / \mathrm{Ni}$ mask. Fig 2 shows the GaN nanopillars after removing the mask. The length and diameter of the NPs were controlled by controlling the etching time and Ni film thickness, respectively. While the etching time determines the length of the NPs, diameter of the NPs depends on the size of the Ni nano-islands. The size of the Ni nano-islands increases with increasing Ni film thickness. Fig 2 (ac) show the SEM images of GaN NPs of lengths 200, $600 \& 900 \mathrm{~nm}$ etched for 60, 90 and $120 \mathrm{~s}$, respectively, using $5 \mathrm{~nm} \mathrm{Ni}$ masked sample. Fig. 2 (d-f) show the NPs with increasing diameter fabricated using 5, 10 and $15 \mathrm{~nm}$ Ni mask, respectively, after etching for $120 \mathrm{sec}$. All the NPs have uniform length and smooth sidewalls irrespective of the etch time and Ni mask size. The average diameter of the NPs measured from the SEM images is 84, 144 and $202 \mathrm{~nm}$ for the 5, 10 and 15 $\mathrm{nm} \mathrm{Ni}$ film masked samples, respectively. The diameter distribution becomes wider with increasing thickness of the Ni film (Fig SI1).

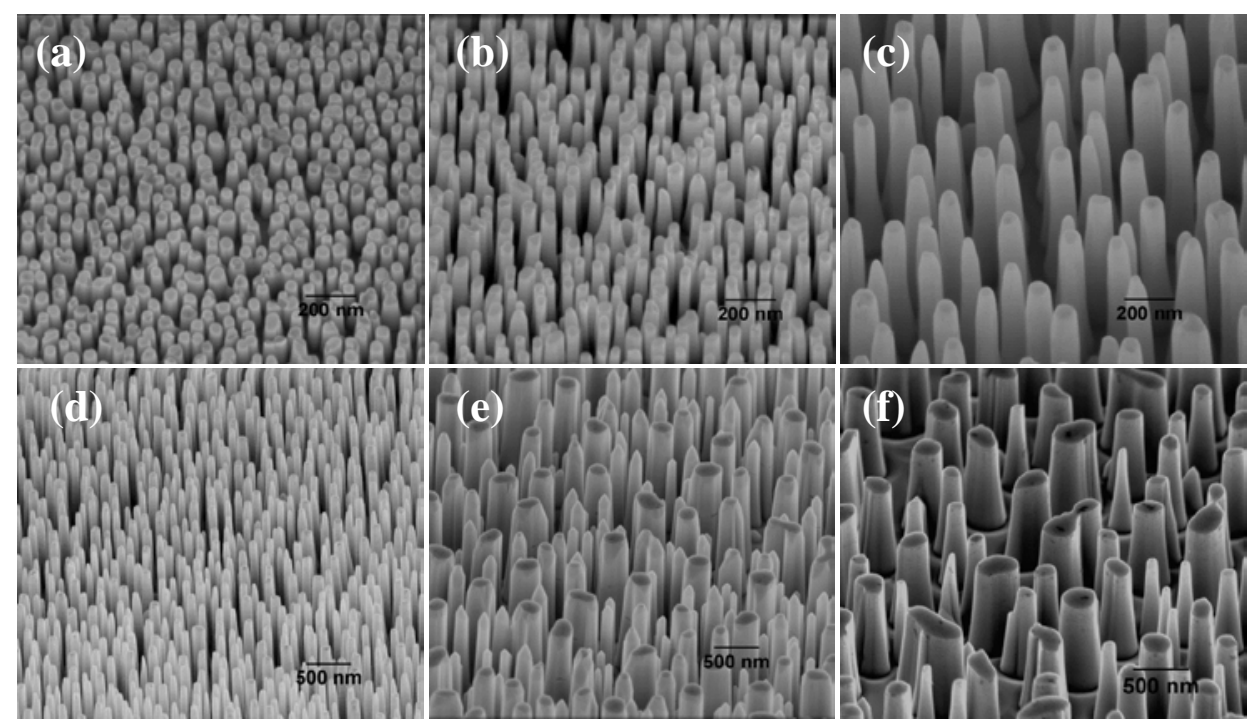

Fig 2. SEM images of the top-down fabricated GaN NPs (images took at $30^{\circ}$ tilt angle) with increasing lengths (a-c) using $5 \mathrm{~nm} \mathrm{Ni}$ mask obtained by varying the etch times from 60 to $120 \mathrm{~s}$ and increasing diameter (d-f) (images took at $52^{0}$ tilt angle)using 5, 10 and $15 \mathrm{~nm}$ Ni mask etched for $120 \mathrm{~s}$. 
Table 1: Sample numbers and the corresponding carrier concentrations

\begin{tabular}{|c|c|}
\hline Sample name & Carrier concentration $\mathbf{( c m}^{-3}$ ) \\
\hline Sample 1(S1) & Undoped \\
\hline Sample 2 (S2) & $1 \times 10^{18}$ \\
\hline Sample 3 (S3) & $5 \times 10^{18}$ \\
\hline Sample 4 (S4) & $1 \times 10^{19}$ \\
\hline
\end{tabular}

(a)

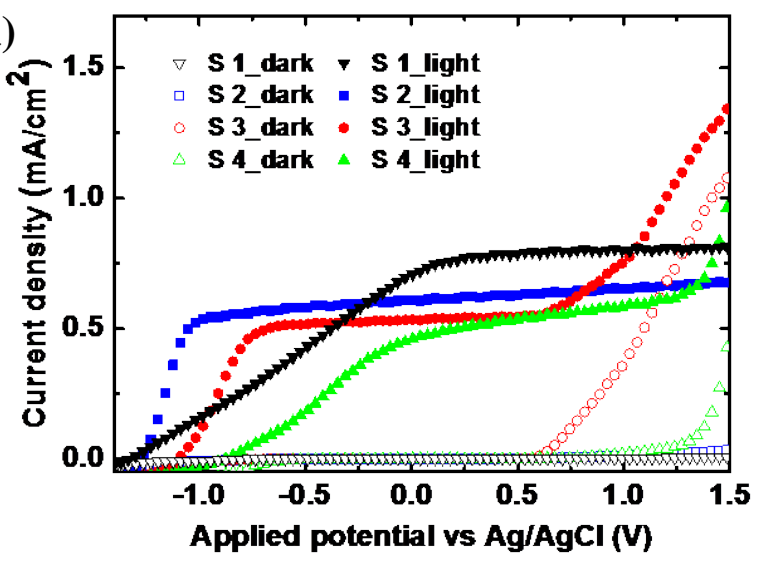

(c)

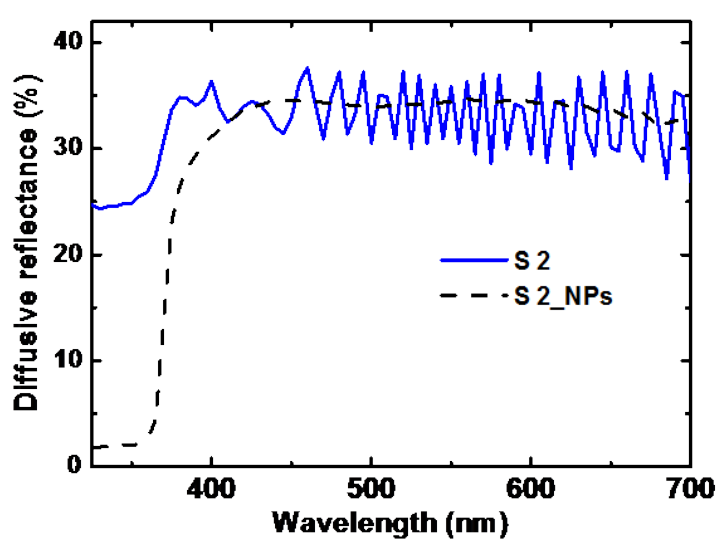

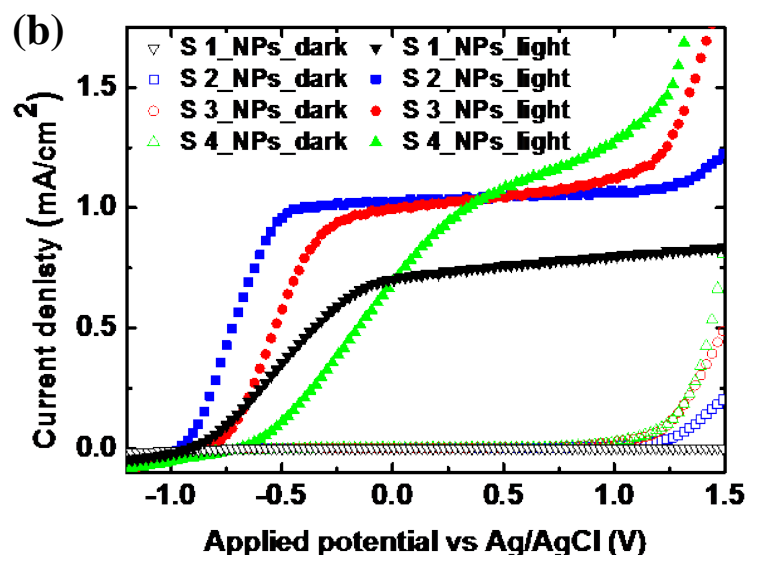

(d)

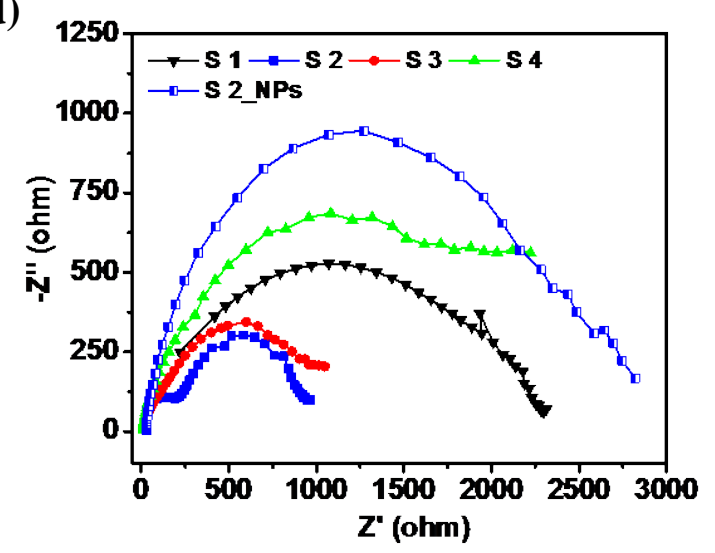

Fig 3. Linear sweep voltammetry of planar GaN photoanodes (a) and GaN NP photoanodes (b), diffuse reflectance spectra (c) and Nyquist plots (d).

Conductivity of the samples plays a critical role in the photoresponse of the photoanodes. As listed in table 1, GaN epilayers were grown with four different carrier concentrations and were used to fabricate NPs using top-down approach. PEC performance of the planar GaN and their respective NPs were evaluated by applying them as a photoanode in a three electrode configuration using a potentiostat. Figure 3(a) shows the PEC performance of GaN planar photoanodes with 
varying carrier concentration. The dark currents for all the samples used in this study, irrespective of the carrier concentration, are insignificantly low. On the other hand, photoresponse of the photoanodes shows a strong dependence on carrier concentration. The onset potential is the lowest for S1 and increases with increasing carrier concentration. However, the photocurrent of S1 increases gradually and reaches a plateau at a much higher potential, indicating a large overpotential is needed for the undoped sample. The lowest overpotential to reach saturation currents is achieved for the photoanode S2 and it increases with increasing carrier concentration. This is attributed to the variation of depletion layer thickness with carrier concentration.[39] In general, the energy bands of the semiconductor are flat in bulk and bend at the surface. The band bending at the surface leads to the formation of charge neutral region (depletion region) near the semiconductor surface. Photogenerated carriers within the depletion zone can be effectively separated due to band bending as compared to those generated in the bulk region due to the flat nature of the bands in the bulk region of the semiconductor. To avoid the inevitable carrier recombination and extract the photogenerated carriers outside the depletion region, a higher overpotential is required. Typically, the thickness of the depletion layer is close to $100 \mathrm{~nm}$ for undoped samples[40] and decreases with increasing carrier concentration (Table SI1). It is noticeable that the overpotential increases with increasing carrier concentration for S2, S3 and S4. However, S1 exhibits the highest overpotential despite the lowest carrier concentration. This is attributed to the poor conductivity of the undoped sample. On the other hand, onset potentials increases with increasing carrier concentration due to decreasing depletion width and the incorporation of structural defects in the epilayer with increasing doping content which may act as recombination centres for the photogenerated carriers.[41] It can be concluded that the lowest doped S2 photoanode has an optimum carrier concentration for lower carrier recombination and higher carrier separation efficiency.

PEC performance of GaN NP photoanodes with different carrier concentrations, fabricated from $120 \mathrm{~s}$ ICP-RIE etching of $5 \mathrm{~nm}$ Ni masked samples, is shown in Fig 3(b). The dark current for NPs photoanodes remains very low. Substantial improvement in saturation photocurrent is noticed for all the doped NP photoanodes compared to the planar ones. The highest saturation current density of around $1.0 \mathrm{~mA} / \mathrm{cm}^{2}$ is measured for all the NP photoanodes. To our knowledge, this is the best reported current density in the literature for GaN NP photoanodes without InGaN quantum wells under one sun light illumination which is also close to the theoretical limit.[42, 43] 
This enhancement can be attributed to the enhanced absorption by the NP photoanodes apart from the increased semiconductor/electrolyte interface area. Fig 3(c) shows the diffuse reflectance spectra of the NP photoanode and its planar counterpart. It clearly shows that NPs absorb most of the light that is incident compared to the planar structures which reflect nearly $24 \%$ of the incident light near the band edge of GaN. This enhancement in absorption contributes to the generation of additional carriers in the NPs that participate in solar water splitting. Further, NPs offer massive semiconductor/interface area compared to the planar structures that result in enhanced depletion region around the semiconductor surface. This increased depletion region leads to the enhancement in carrier separation and efficiently drives the holes to the semiconductor-electrolyte interface.

Further, the onset and saturation potential shifts slightly towards higher potential for all the NP photoanodes irrespective of the carrier concentration with respect to the planar photoanodes. This anodic shift is attributed to the surface damage caused to the NP surface during plasma etching.[38] The surface defects act as traps for photogenerated charge carriers at the NP surface and therefore higher potential is required to extract the photogenerated carriers that are trapped in these defects. This leads to higher onset potential for NP photoanodes compared to the planar photoanodes. Electrochemical impedance measurements were carried out to understand the charge transfer kinetics between semiconductor and electrolyte as shown in Fig 3(d). The diameter of the half circles in the Nyquist plot is linked to the charge transfer resistance between semiconductor surface and electrolyte. Typically, the smaller the diameter the lower is the charge transfer resistance. The Nyquist plots indicate that the charge transfer resistance is increased for NPs photoanodes compared to the planar photoanodes. The increased charge transfer resistance is due to plasma etching damage for NP photoanodes and contributes to anodic shift in the onset potential and over potentials. Among the planar photoanodes, S2 exhibits the lowest charge transfer resistance. For photoanodes with higher carrier concentration, structural defects created by high doping and reduced depletion region causes increased charge transfer resistance between the semiconductor surface and electrolyte. 

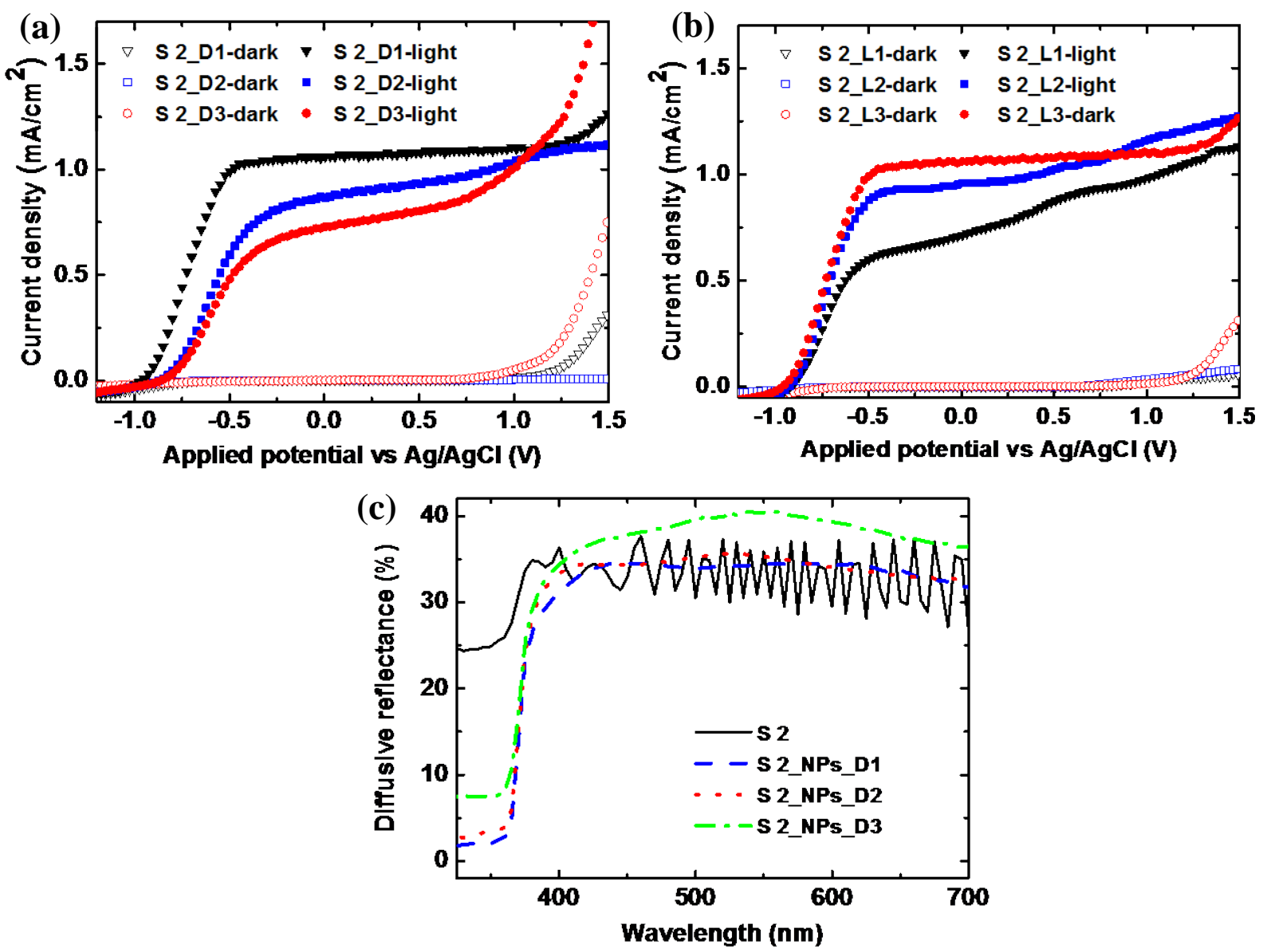

Fig 4. Linear sweep voltammetry of GaN NP photoanodes of varying diameters (a), varying lengths (b) and diffuse reflectance of NP photoanodes with varying diameters (c).

To investigate the effect of diameter and length on the PEC performance of NP photoanodes, NPs with different diameters and lengths are fabricated using S2. The diameters of the NP photoanodes are classified as a small (D1), medium (D2) and large (D3) fabricated from 5, 10 and $15 \mathrm{~nm}$ Ni masked samples, respectively. The diameter of the NPs is increased while the density is decreased with increasing Ni mask thickness under the same processing conditions as observed in Fig 2 (d) to (f). As shown in Fig 4(a), smallest diameter NP photoanodes exhibit the highest saturation photocurrent density and it decreases with increasing NP diameter. Increasing the diameter of NPs results in the reduction of semiconductor/electrolyte interface area. Consequently the depletion region is reduced with increasing NP diameter and causes inefficient separation of photogenerated carriers which could in turn contribute to the decreased saturation current density. In order to understand the effect of NP diameter on light absorption, diffusive 
reflectance measurements were carried out and the results are shown in Fig 4(c). Reflection losses for NPs are significantly lower than epilayers irrespective of the diameters of the NPs. However, the reflection losses slightly increased with increasing diameter of the NPs. Higher reflection losses leads to the reduced light absorptions and carrier generation. Further, from Fig 4(a), it can also be observed that there is a slight change in onset potential with NP diameter. Due to the presence of surface band bending, the NPs could be either completely or partially depleted depending on the diameter of NPs for a given carrier concentration. The average diameter of the nanopillars is 84, 144 and $202 \mathrm{~nm}$ for D1, D2 and D3 NP photoanodes, respectively. The estimated depletion layer width in one dimension is $\sim 24.5 \mathrm{~nm}$ for $1 \times 10^{18} \mathrm{~cm}^{-3}$ carrier concentration (Table SI1).[44] For a given carrier concentration, the width of the depletion region is constant. Therefore, width of the flat band region increases with increasing NP diameter [45]. The deduced thickness of the flat band region is 35, 95 and $153 \mathrm{~nm}$ for D1, D2 and D3 NP photoanodes, respectively. As a consequence, recombination of the photogenerated carriers becomes severe with increasing diameter of the NPs which could in turn contribute to increased onset potential with increasing NP diameter. However, the role of depletion region may become insignificant compared to the flat band region beyond certain NP diameter.[45]

Figure 4 (b) shows the influence of NP length on the current density of NP photoanodes under dark and light illumination. NPs fabricated from $5 \mathrm{~nm}$ Ni mask pattern were chosen for this study and the lengths of the NPs were 200, 500 and $900 \mathrm{~nm}$ labelled as short (L1), medium (L2) and long (L3) NP photoanodes, respectively. Since the NPs diameter is fixed while studying the influence of NPs length on PEC performance, insignificant changes in onset potential is observed regardless of the NP length. The onset potential is dependent on the depletion layer width that varies only with the diameter and doping concentration. On the other hand the saturation current is improved with increasing NP length. NPs/electrolyte interface area is estimated to be linearly dependent on the length of the NPs which contributes to increase depletion region volume with increasing NPs length.[44] The increase in depletion region volume leads to increased saturation level of the photo current. It is also expected that a certain minimum length of NPs is necessary to mitigate the optical reflection losses.[46] This could be another possible reason for the reduction in photocurrent density with reducing length of the NPs. 

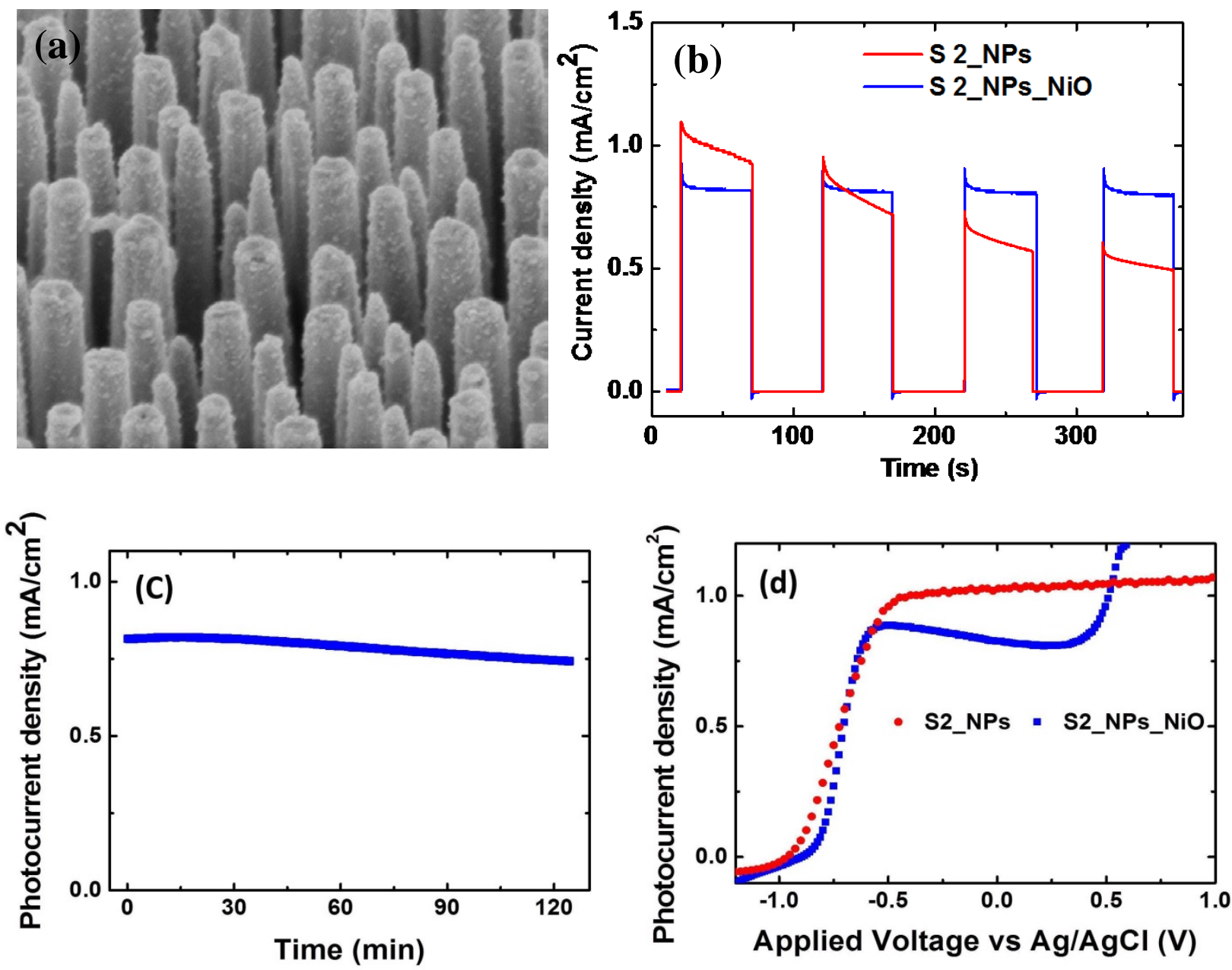

Fig 5. (a) SEM image of NiO co-catalyst deposited GaN NPs, (b) short term photostability of NP photoanodes with and without $\mathrm{NiO}$ co-catalyst and (c) long term photostability of NiO coated NP photoanodes under AM1.5 light illumination at $0 \mathrm{~V}$ vs. Ag/AgCl in $1 \mathrm{M} \mathrm{NaOH}$ electrolyte and (d) linear sweep voltammetry of GaN NP photoanodes with and without $\mathrm{NiO}$ co-catalyst

It is well known that c-plane of GaN is chemically stable compared to the m-plane. [47, 48] When the NP photoanodes are dipped in electrolyte solution the m-plane is exposed to the electrolyte and results in photocurrent degradation with time. In order to improve the photostability of the NPs, NiO co-catalyst was coated around NP surface. From the SEM image shown in Fig 5(a), it can be observed that $\mathrm{NiO}$ co-catalyst nanoparticles are uniformly decorated around the NPs. The transient photocurrent curves for NP photoanodes with and without NiO co-catalyst are shown in Fig 5(b). The photocurrent density became relatively stable after NiO coating. The improved stability can be attributed to discontinuities in the bands between $\mathrm{GaN}$ and $\mathrm{NiO}$ [36, 41, 49]. NiO energy band positions are located at higher energy relative to the GaN energy bands (Fig. 
SI2). As a consequence, the migration/extraction of photogenerated holes from $\mathrm{GaN}$ to $\mathrm{NiO}$ is enhanced, thereby significantly reducing the number of holes accumulated at the NPs/electrolyte interface. The reduced number of accumulated holes at the interface thus leads to reduced oxidative etching of the NPs surface. The long term stability of the NiO coated NP photoanodes is shown in Fig 5(c). Slight drop in the photocurrent density is noticed after $2 \mathrm{hr}$ of illumination. Figure 5(d) shows the linear sweep voltammetry of NP photoanodes with and without NiO catalyst under solar illumination. Small drop in the photocurrent density is observed for NiO coated NP photoanodes which could be due to the parasitic light absorption by the $\mathrm{NiO}$ nanoparticles. Further, a sharp rise in photocurrent density is observed for $\mathrm{NiO}$ coated GaN NP photoanodes beyond $0.5 \mathrm{~V}$. This voltage is close to the reported OER potential of $\mathrm{NiO}$ catalyst[50] and hence the sharp raise of photocurrent density beyond this value can be attributed to the electrochemical catalytic activity of $\mathrm{NiO}$.

\section{Conclusions}

To conclude, GaN NP photoanodes were fabricated using the top-down approach and exhibited substantial enhancement in the PEC performance compared to their planar counterparts. This enhancement is found to be strongly influenced by the carrier concentration and nanopillars dimensions. The photocurrent of n-doped NP photoanodes can reach up to $1 \mathrm{~mA} / \mathrm{cm}^{2}$, which is close to the theoretical limit, owing to their enhanced optical absorption and increased semiconductor/electrolyte interface. An anodic potential shift for the NP photoanodes is observed with increasing carrier concentration due to decreased depletion layer thickness and increased structural defects. Photocurrent density is reduced with increasing diameter and reducing length of the NPs. Reduction in optical absorption and depletion region can also contribute to the drop in the photocurrent density. Furthermore, increasing the diameter of the NP photoanodes for a given carrier concentration is found to cause an anodic shift of the onset potential due to the increased flat band region of the NPs. Finally, photodegradation of the NP photoanodes is substantially reduced by decorating the GaN NPs with NiO co-catalysts.

\section{Acknowledgements}

We acknowledge the Australian Research Council for providing financial support and the Australian National Fabrication Facility (ANFF) for providing access to the epitaxial growth and fabrication facilities. 


\section{References}

[1] Lewis N S and Nocera D G 2006 Powering the planet: Chemical challenges in solar energy utilization Proceedings of the National Academy of Sciences 103 15729-35

[2] Kamat P V and Christians J A 2015 Solar Cells versus Solar Fuels: Two Different Outcomes The Journal of Physical Chemistry Letters 6 1917-8

[3] Walter M G, Warren E L, McKone J R, Boettcher S W, Mi Q, Santori E A and Lewis N S 2010 Solar Water Splitting Cells Chemical Reviews 110 6446-73

[4] AlOtaibi B, Fan S, Vanka S, Kibria M G and Mi Z 2015 A Metal-Nitride Nanowire DualPhotoelectrode Device for Unassisted Solar-to-Hydrogen Conversion under Parallel Illumination Nano letters 15 6821-8

[5] Fan S, AlOtaibi B, Woo S Y, Wang Y, Botton G A and Mi Z 2015 High Efficiency Solarto-Hydrogen Conversion on a Monolithically Integrated InGaN/GaN/Si Adaptive Tunnel Junction Photocathode Nano letters 15 2721-6

[6] Zhao S, Nguyen H P T, Kibria M G and Mi Z 2015 III-Nitride nanowire optoelectronics Progress in Quantum Electronics 44 14-68

[7] Kibria M G and Mi Z 2016 Artificial photosynthesis using metal/nonmetal-nitride semiconductors: current status, prospects, and challenges Journal of Materials Chemistry A 4 2801-20

[8] Zakutayev A 2016 Design of nitride semiconductors for solar energy conversion Journal of Materials Chemistry A 4 6742-54

[9] Moses P G and Van de Walle C G 2010 Band bowing and band alignment in InGaN alloys Applied Physics Letters 96021908

[10] Reddy N P, Naureen S, Mokkapati S, Vora K, Shahid N, Karouta F, Tan H H and Jagadish C 2016 Enhanced luminescence from GaN nanopillar arrays fabricated using a top-down process Nanotechnology 27065304

[11] Spurgeon J M, Atwater H A and Lewis N S 2008 A comparison between the behavior of nanorod array and planar Cd(Se, Te) photoelectrodes J Phys Chem C 112 6186-93

[12] Xiao F-X, Miao J, Tao H B, Hung S-F, Wang H-Y, Yang H B, Chen J, Chen R and Liu B 2015 One-Dimensional Hybrid Nanostructures for Heterogeneous Photocatalysis and Photoelectrocatalysis Small 11 2115-31 
[13] Chen H M, Chen C K, Liu R-S, Zhang L, Zhang J and Wilkinson D P 2012 Nanoarchitecture and material designs for water splitting photoelectrodes Chemical Society Reviews 41 5654-71

[14] Jung H S, Hong Y J, Li Y, Cho J, Kim Y-J and Yi G-C 2008 Photocatalysis Using GaN Nanowires ACS Nano 2 637-42

[15] Kato H, Asakura K and Kudo A 2003 Highly Efficient Water Splitting into H2 and O2 over Lanthanum-Doped NaTaO3 Photocatalysts with High Crystallinity and Surface Nanostructure Journal of the American Chemical Society 125 3082-9

[16] Bergbauer W, Strassburg M, Kolper C, Linder N, Roder C, Lahnemann J, Trampert A, Fundling S, Li S F, Wehmann H H and Waag A 2010 Continuous-flux MOVPE growth of position-controlled $\mathrm{N}$-face $\mathrm{GaN}$ nanorods and embedded InGaN quantum wells Nanotechnology 21305201

[17] Lin Y T, Yeh T W and Dapkus P D 2012 Mechanism of selective area growth of GaN nanorods by pulsed mode metalorganic chemical vapor deposition Nanotechnology 23 465601

[18] Paramanik D, Motayed A, Aluri G S, Ha J-Y, Krylyuk S, Davydov A V, King M, McLaughlin S, Gupta S and Cramer H 2012 Formation of large-area GaN nanostructures with controlled geometry and morphology using top-down fabrication scheme Journal of Vacuum Science \& Technology B 30052202

[19] Wang Y D, Chua S J, Tripathy S, Sander M S, Chen P and Fonstad C G 2005 High optical quality GaN nanopillar arrays Applied Physics Letters 86071917

[20] Zhuang Z, Guo X, Zhang G, Liu B, Zhang R, Zhi T, Tao T, Ge H, Ren F, Xie Z and Zheng Y 2013 Large-scale fabrication and luminescence properties of GaN nanostructures by a soft UV-curing nanoimprint lithography Nanotechnology 24405303

[21] Hu Y, Hao Z, Lai W, Geng C, Luo Y and Yan Q 2015 Nano-fabrication and related optical properties of InGaN/GaN nanopillars Nanotechnology 26075302

[22] Bergbauer W, Strassburg M, Kölper C, Linder N, Roder C, Lähnemann J, Trampert A, Fündling S, Li S F, Wehmann H H and Waag A 2011 N-face GaN nanorods: Continuousflux MOVPE growth and morphological properties Journal of Crystal Growth 315 164-7 
[23] Chiu C H, Lu T C, Huang H W, Lai C F, Kao C C, Chu J T, Yu C C, Kuo H C, Wang S C, Lin C F and Hsueh T H 2007 Fabrication of InGaN/GaN nanorod light-emitting diodes with self-assembled Ni metal islands Nanotechnology 18445201

[24] Zhu J, Wang L, Zhang S, Wang H, Zhao D, Zhu J, Liu Z, Jiang D and Yang H 2010 The fabrication of GaN-based nanopillar light-emitting diodes Journal of Applied Physics 108 074302

[25] Ebaid M, Kang J-H, Lim S-H, Ha J-S, Lee J K, Cho Y-H and Ryu S-W 2015 Enhanced solar hydrogen generation of high density, high aspect ratio, coaxial InGaN/GaN multiquantum well nanowires Nano Energy 12 215-23

[26] Caccamo L, Hartmann J, Fabrega C, Estrade S, Lilienkamp G, Prades J D, Hoffmann M W G, Ledig J, Wagner A, Wang X, Lopez-Conesa L, Peiro F, Rebled J M, Wehmann H H, Daum W, Shen H and Waag A 2014 Band Engineered Epitaxial 3D GaN-InGaN CoreShell Rod Arrays as an Advanced Photoanode for Visible-Light-Driven Water Splitting Acs Appl Mater Inter 6 2235-40

[27] AlOtaibi B, Nguyen H P, Zhao S, Kibria M G, Fan S and Mi Z 2013 Highly stable photoelectrochemical water splitting and hydrogen generation using a double-band InGaN/GaN core/shell nanowire photoanode Nano letters 13 4356-61

[28] Ebaid M, Kang J-H, Lim S-H, Cho Y-H and Ryu S-W 2015 Towards highly efficient photoanodes: the role of carrier dynamics on the photoelectrochemical performance of InGaN/GaN multiple quantum well coaxial nanowires RSC Advances 5 23303-10

[29] AlOtaibi B, Harati M, Fan S, Zhao S, Nguyen H P, Kibria M G and Mi Z 2013 High efficiency photoelectrochemical water splitting and hydrogen generation using $\mathrm{GaN}$ nanowire photoelectrode Nanotechnology 24175401

[30] Wallys J, Hoffmann S, Furtmayr F, Teubert J and Eickhoff M 2012 Electrochemical properties of GaN nanowire electrodes--influence of doping and control by external bias Nanotechnology 23165701

[31] Hwang J S, Liu T Y, Chattopadhyay S, Hsu G M, Basilio A M, Chen H W, Hsu Y K, Tu W H, Lin Y G, Chen K H, Li C C, Wang S B, Chen H Y and Chen L C 2013 Growth of beta-Ga2O3 and GaN nanowires on GaN for photoelectrochemical hydrogen generation Nanotechnology 24055401 
[32] Kibria M G, Chowdhury F A, Zhao S, AlOtaibi B, Trudeau M L, Guo H and Mi Z 2015 Visible light-driven efficient overall water splitting using p-type metal-nitride nanowire arrays Nature communications 66797

[33] Kibria M G, Zhao S, Chowdhury F A, Wang Q, Nguyen H P, Trudeau M L, Guo H and Mi Z 2014 Tuning the surface Fermi level on p-type gallium nitride nanowires for efficient overall water splitting Nature communications 53825

[34] Wang D, Pierre A, Kibria M G, Cui K, Han X, Bevan K H, Guo H, Paradis S, Hakima A R and Mi Z 2011 Wafer-level photocatalytic water splitting on GaN nanowire arrays grown by molecular beam epitaxy Nano letters 11 2353-7

[35] Phivilay S P, Roberts C A, Puretzky A A, Domen K and Wachs I E 2013 Fundamental Bulk/Surface Structure-Photoactivity Relationships of Supported (Rh2-yCryO3)/GaN Photocatalysts The Journal of Physical Chemistry Letters 4 3719-24

[36] Benton J, Bai J and Wang T 2013 Significantly enhanced performance of an InGaN/GaN nanostructure based photo-electrode for solar power hydrogen generation Applied Physics Letters 103133904

[37] Tseng W J, van Dorp D H, Lieten R R, Mehta B, Vereecken P M and Borghs G 2013 Enhanced Photocatalytic Activity of Nanoroughened GaN by Dry Etching ECS Electrochemistry Letters 2 H51-H3

[38] Tseng W-J, van Dorp D H, Lieten R R, Vereecken P M, Langer R and Borghs G 2014 Impact of Plasma-Induced Surface Damage on the Photoelectrochemical Properties of GaN Pillars Fabricated by Dry Etching The Journal of Physical Chemistry C 118 11261-6

[39] Richter T, Meijers H L, Calarco R and Marso M 2008 Doping concentration of GaN nanowires determined by opto-electrical measurements Nano letters 8 3056-9

[40] Iwaki Y, Ono M, Yamaguchi K, Kusakabe K, Fujii K and Ohkawa K 2008 Nitride photocatalyst to generate hydrogen gas from water physica status solidi (c) 5 2349-51

[41] Kang J-H, Kim S H, Ebaid M, Lee J K and Ryu S-W 2014 Efficient photoelectrochemical water splitting by a doping-controlled GaN photoanode coated with $\mathrm{NiO}$ cocatalyst Acta Materialia 79 188-93

[42] Zhebo Chen T G D, Huyen N. Dinh, Kazunari Domen, Keith Emery, Arnold J. Forman, Nicolas Gaillard, Roxanne Garland, Clemens Heske, Thomas F. Jaramillo, Alan Kleiman- 
Shwarsctein, Eric Miller , Kazuhiro Takanabe, John TurnerShow 2013 Photoelectrochemical Water Splitting ed H N D Zhebo Chen, Eric Miller Springer)

[43] Shockley W and Queisser H J 1961 Detailed Balance Limit of Efficiency of p-n Junction Solar Cells Journal of Applied Physics 32 510-9

[44] Kornienko N, Gibson N A, Zhang H, Eaton S W, Yu Y, Aloni S, Leone S R and Yang P 2016 Growth and Photoelectrochemical Energy Conversion of Wurtzite Indium Phosphide Nanowire Arrays ACS Nano 10 5525-35

[45] Calarco R, Marso M, Richter T, Aykanat A I, Meijers R, A V D H, Stoica T and Luth H 2005 Size-dependent photoconductivity in MBE-grown GaN-nanowires Nano letters 5 $981-4$

[46] Jung J-Y, Um H-D, Jee S-W, Park K-T, Bang J H and Lee J-H 2013 Optimal design for antireflective Si nanowire solar cells Solar Energy Materials and Solar Cells 112 84-90

[47] Lai Y-Y, Hsu S-C, Chang H-S, Wu Y S, Chen C-H, Chen L-Y and Cheng Y-J 2016 The study of wet etching on GaN surface by potassium hydroxide solution Research on Chemical Intermediates 1-10

[48] Li Q, Westlake K R, Crawford M H, Lee S R, Koleske D D, Figiel J J, Cross K C, Fathololoumi S, Mi Z and Wang G T 2011 Optical performance of top-down fabricated InGaN/GaN nanorod light emitting diode arrays Opt. Express 19 25528-34

[49] Waki I, Cohen D, Lal R, Mishra U, DenBaars S P and Nakamura S 2007 Direct water photoelectrolysis with patterned n-GaN Applied Physics Letters 91093519

[50] Bernicke M, Eckhardt B, Lippitz A, Ortel E, Bernsmeier D, Schmack R and Kraehnert R 2016 Synthesis and OER activity of $\mathrm{NiO}$ coatings with micelle-templated mesopore structure ChemistrySelect 1 482-9 


\section{Supporting Information}

\section{Improved Photoelectrochemical Performance of GaN Nanopillar Photoanodes}

Parvathala Reddy Narangari ${ }^{\mathrm{a}^{*}}$, Siva Krishna Karuturi $\mathrm{a}^{\mathrm{a}^{*}}$, Mykhaylo Lysevych ${ }^{\mathrm{b}}$, Hark Hoe Tan ${ }^{\mathrm{a}}$, and Chennupati Jagadish ${ }^{\mathrm{a}}$

${ }^{a}$ Department of Electronic Materials Engineering, Research School of Physics and Engineering, The Australian National University, Canberra, ACT 2601, Australia

${ }^{b}$ Australian National Fabrication Facility, Research School of Physics and Engineering, The Australian National University, Canberra, ACT 2601, Australia

Corresponding author: parvathala.narangari@anu.edu.au; siva.karuturi@anu.edu.au

\section{Calculation of depletion layer width:}

$$
\begin{gathered}
\text { Depletion layer width }(W)=\sqrt{\frac{\epsilon_{0} \epsilon \Delta V}{e N_{d}}} \\
\text { Where } \epsilon_{0}=\text { Vacuum permittivity } \\
\epsilon=\text { dielectric constant of } \mathrm{GaN}
\end{gathered}
$$

$\Delta \mathrm{V}=$ potential difference between $\mathrm{GaN}$ and the redox couple in the solution

$$
\begin{gathered}
\mathrm{e}=\text { elementary charge } \\
\mathrm{N}_{\mathrm{d}}=\text { Carrier concentration }
\end{gathered}
$$

Table SI1: Estimated depletion layer width with different carrier concentrations in GaN epilayers

\begin{tabular}{|c|c|}
\hline Carrier concentration $\left(\mathrm{cm}^{-3}\right)$ & Depletion layer width (nm) \\
\hline $1 \mathrm{X} 10^{18}$ & 24.5 \\
\hline $5 \mathrm{X} 10^{18}$ & 11 \\
\hline $1 \mathrm{X} 10^{19}$ & 7.7 \\
\hline
\end{tabular}


(a)

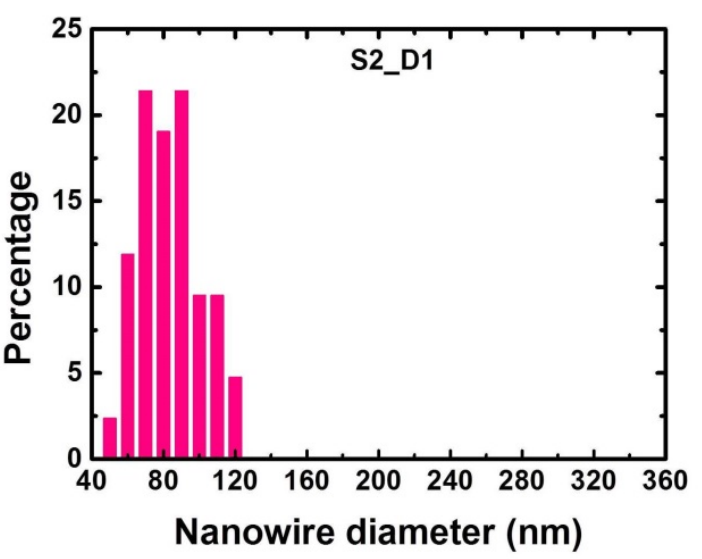

(b)

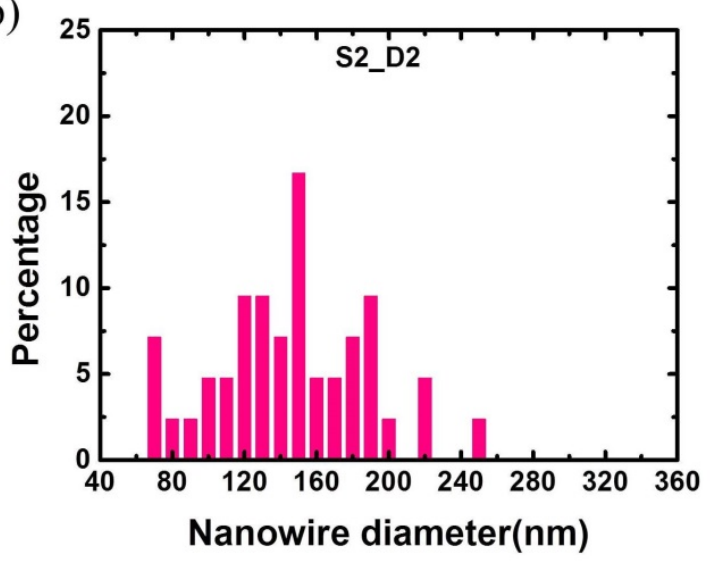

(c)

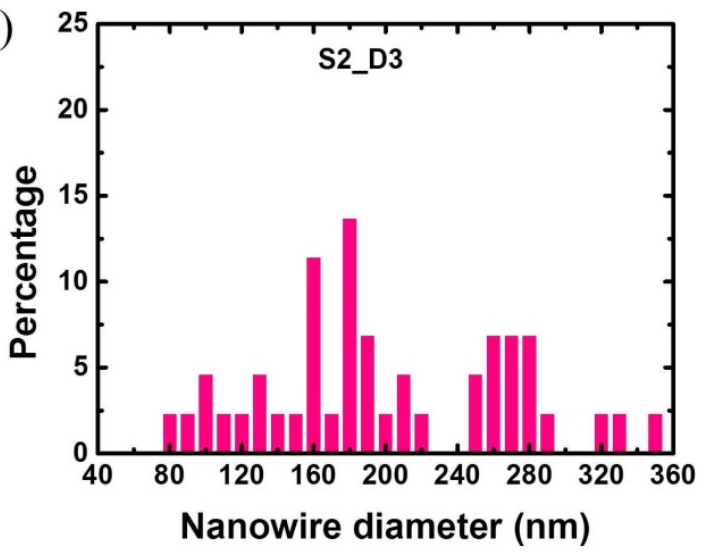

Figure SI1: Distribution of nanopillars diameters fabricated using (a) 5, (b) 10 and (c) 15nm Ni film.

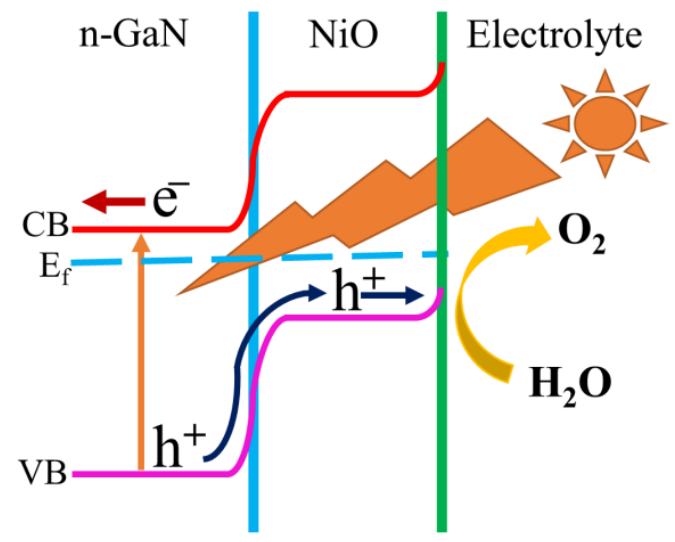

Figure SI2: Schematic of energy band diagram of GaN/NiO/electrolyte and electron and hole transfer at the material interfaces (CB: conduction band, VB: valency band and $E_{f}$ : Fermi level). 